\title{
The spin-1/2 anisotropic Heisenberg-chain in longitudinal and transversal magnetic fields: a DMRG study.
}

\author{
Felicien Capraro and Claudius Gros \\ Universität des Saarlandes, Fakultät 7.1, Postfach 1511 50, 66041 Saarbrücken, Germany \\ the date of receipt and acceptance should be inserted later
}

\begin{abstract}
Using the density matrix renormalization group technique, we evaluate the low-energy spectrum (ground state and first excited states) of the anisotropic antiferromagnetic spin-one-half chain under magnetic fields. We study both homogeneous longitudinal and transversal fields as well as the influence of a transversal staggered field on opening of a spin-gap. We find that only a staggered transversal field opens a substantial gap.
\end{abstract}

PACS. $\quad$ 75.10.Jm Quantized spin models

75.50.Ee Studies of specific magnetic materials ( Antiferromagnetic)

\section{Introduction}

Recently, the properties of low-dimensional quantum spin systems in longitudinal, transversal and/or staggered magnetic fields have become of considerable interest. To give an example, the two-dimensional compound $\mathrm{SrCu}_{2}\left(\mathrm{BO}_{3}\right)_{2}$ is a realization of the Shastry-Sutherland model [1], close to quantum-criticality, with a spin gap of $31 \mathrm{~K}$ which shows magnetization plateaus in an external field [2].

Most spin- $1 / 2$ systems show little anisotropies in the magnetic exchange. The discovery [3] that low-dimensional magnetic excitations in the rare-earth compound $\mathrm{Yb}_{4} \mathrm{As}_{3}$ can explain the large linear specific heat coefficient $\gamma$ in this low-carrier half-metal [, 1,5 opens the possibility to study in deeper detail the properties of a rare-earth quantum-spin-chain-system with its enhanced magnetic anisotropies [6,7].

Inelastic neutron scattering experiments [8] on $\mathrm{Yb}_{4} \mathrm{As}_{3}$ found a gap to (all) magnetic excitations opening in the presence of an external magnetic field, confirming a prediction [3] by Schmidt et al based on an interpretation of previous specific heat data [9]. Several proposals have been made in order to explain this very unusual behavior [10]. The first model [3] is based on inter-chain interactions. The second model [11] is based on the observation that a staggered Dzyaloshinsky-Moriya (DM) interaction, which generates an effective staggered g-tensor, is allowed [6, 7] in the $4 f$-compound $\mathrm{Yb}_{4} \mathrm{As}_{3}$. It is know that a staggered g-tensor leads to a gap in an external field [12, 13]. The third model [10], based on a mean-field analyze of the anisotropic spin-chain, proposes that a gap opens in the presence of a uniform transversal magnetic field.

Here we will analysis the two latter proposals by a systematic DMRG-studies of the relevant models. We find that only the effective staggered g-factor-model is able to explain the field-dependent opening of a spin-gap in $\mathrm{Yb}_{4} \mathrm{As}_{3}$.

\subsection{Model and method}

The magnetic properties of $\mathrm{Yb}_{4} \mathrm{As}_{3}$, in the absence of an external magnetic field, are well described by an antiferromagnetic Heisenberg spin-1/2 chain. Switching on the external magnetic field, experimental data shows the opening of a gap in the low energy excitation spectrum. However, the standard Heisenberg model in an applied field remains gapless from zero magnetic field up to the saturation magnetization. The anisotropic Heisenberg model

$$
\begin{aligned}
H & =\sum_{i=1}^{L-1} J_{x y}\left(S_{i}^{x} S_{i+1}^{x}+S_{i}^{y} S_{i+1}^{y}\right)+J_{z} S_{i}^{z} S_{i+1}^{z} \\
& -\sum_{i=1}^{L} h_{x} S_{i}^{x}+\sum_{i=1}^{L} h_{x}^{\text {stag }}(-1)^{i} S_{i}^{x}-\sum_{i=1}^{L} h_{z} S_{i}^{z}
\end{aligned}
$$

with an uniform transversal field $h_{x}$, a staggered transversal field $h_{x}^{\text {stag }}$ and a longitudinal field $h_{z}$ incorporates all features proposed [10,11] to be relevant for $\mathrm{Yb}_{4} \mathrm{As}_{3}$. The magnetic fields appearing in (1) include the gyromagnetic g-factors.

The staggered transversal field in (1) is induced by a staggered Dzyaloshinsky-Moriya interaction given by the term $\sum_{i}(-1)^{i} \mathbf{D} \cdot\left(\mathbf{S}_{i} \times \mathbf{S}_{i+1}\right)$. Setting $D=|\mathbf{D}|=J_{z} \sin (2 \theta)$ the DM-term can be eliminated [12, 14] by a rotation around D by an angle $\theta$ leading to $h_{x}^{\text {stag }}=\sin (\theta) h_{z}$, which can be interpreted as an effective staggered g-tensor.

We have simulated the anisotropic Heisenberg model (17) using the Density Matrix Renormalization Group (DMRG) technique 15. We have, in general, investigated carefully the dependence of the results on the number $m$ of states kept in the DMRG calculations. If not stated otherwise, we have used open boundary conditions, the finite-systemsize algorithm and extrapolated the finite-size data to the thermodynamic limit, setting $J_{x y} \equiv 1$ in (1). 


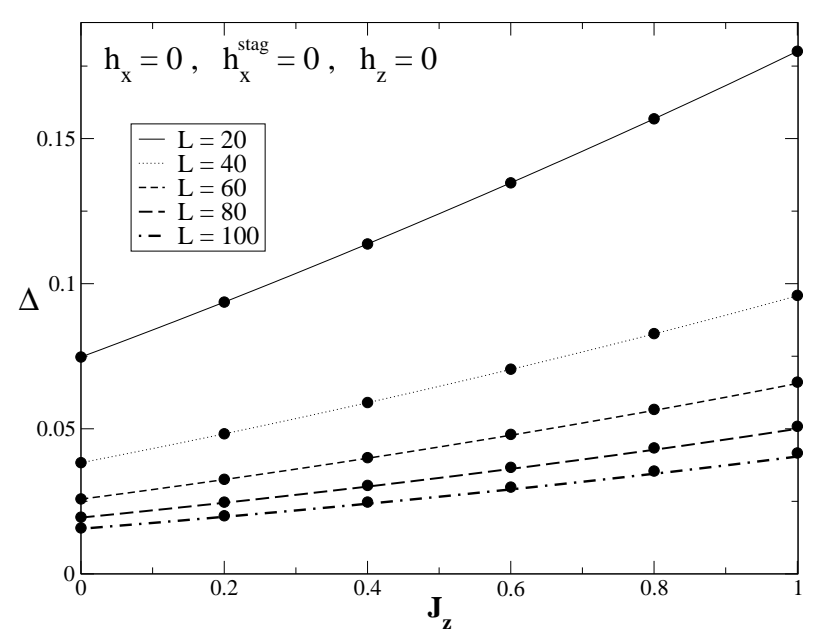

Fig. 1. Comparison between DMRG results (filled circles) for the finite-size gap $\Delta(L)$, using $m=50$ states, and the BetheAnsatz results (lines). The gap is given as a function of the anisotropy $J_{z}$ in the absence of external magnetic fields.

\subsection{Comparison of DMRG and Bethe-Ansatz results}

In order to verify the accuracy of our approach for the excitation energies we use the Bethe-Ansatz equations 18 , 19] to evaluate the gap $\Delta(L)$ for finite chains with size $L$,

$$
\Delta(L)=E_{1}(L)-E_{0}(L),
$$

for the anisotropic antiferromagnetic Heisenberg chain and then compare it with our DMRG simulations. In Fig. 1 we plot the finite-size gap obtained by using DMRG versus the anisotropy $\left(0 \leq J_{z} \leq 1\right)$, the lines correspond to the exact finite-size gap obtained using Bethe-Ansatz. Although in this regime the gap in the thermodynamic limit is equal to zero, the correspondence between our DMRG simulations for the gap for finite system sizes with the Bethe-Ansatz results is very accurate.

\section{Homogeneous transversal magnetic field}

Uimin et al proposed, by a mean-field calculation 10], that a gap opens for the spin- $1 / 2$ anisotropic Heisenberg chain

$$
\begin{array}{r}
H=\sum_{i=1}^{L-1} J_{x y}\left(S_{i}^{x} S_{i+1}^{x}+S_{i}^{y} S_{i+1}^{y}\right) \\
+J_{z} S_{i}^{z} S_{i+1}^{z} \\
-h_{x} \sum_{i=1}^{L} S_{i}^{x}
\end{array}
$$

in the presence of a homogeneous longitudinal field $h_{x}$. This mean-field result would then imply that no further magnetic anisotropies would be needed to explain the experimentally observed spin-gap of $\mathrm{Yb}_{4} \mathrm{As}_{3}$.

In spin-wave theory Uimin et al found [10] for the model (2) a gap $\Delta_{S W T}$ consisting of two branches:

$$
\Delta_{S W T}=\min \left(\Delta_{1}, \Delta_{2}\right)
$$

with $\Delta_{1}=h_{x}$ and

$$
\Delta_{2}=\sqrt{\frac{2}{3}\left(\frac{1-J_{z}}{2+J z}\right)\left(\left(2+J_{z}\right)^{2}-\left(\frac{3 h_{x}}{2}\right)^{2}\right)} .
$$

Up to a certain magnetic field the value of the gap is almost linear and above this value the gap starts to close itself following a quadratic form, compare Fig. 14 and Fig. 年.

The physical reasoning for the gap present in the meanfield results reviewed above is the following: neglecting the $S_{i}^{x} S_{i+1}^{x}$ coupling term in the Hamiltonian (2) it becomes identical to the Ising model in a transversal field, which has a two-fold degenerate ground-state and a gap. It has been argued 16, 17, that this reasoning remains valid also for (2).

We compute the energy gap $\Delta(L)$ using the DMRG. We calculate the ground state and the lowest excited states energies $E_{i}(i=0,1,2)$ and form the following energy difference $\Delta_{1}=E_{1}-E_{0}$ and $\Delta_{2}=E_{2}-E_{0}$ respectively between the first and the second excited state with the ground state energy. Fig. 2 and Fig. 3 show the behavior of $\Delta_{i}(L)$ with the transverse magnetic field $h_{x}$ for $J_{z}=0.75,0.9706$.

In the thermodynamic limit the first energy difference $\Delta_{1} \rightarrow 0$ in the low magnetic field regime $\left(h_{x} \ll\right.$ 2 ). This behavior is in agreement with the fact that the GS is doubly degenerated in this regime when $L \rightarrow \infty$. Thus to estimate the eventually system-gap in this regime we calculate $\Delta_{2} . \Delta_{2}$ shows a more monotonous behavior. For fixed $h_{x}, \Delta_{2}(L)$ is decreasing with the system-size $L\left(\Delta_{2}(100) \leq 0.04\right)$. Fig. 1 and Fig. 5 show the extrapolate gap for the thermodynamic limit with $J_{x y}=1$ and $J_{z}=0.75,0.9706$. We have evaluated also the induced uniform magnetization $M_{x}$, which we present in Fig. 6 for $J_{z}=0.25$ and $J_{z}=0.9706$ (value which corresponds to the small anisotropies in $x y$-plane appropriate for $\mathrm{Yb}_{4} \mathrm{As}_{3}[3]$ ).

We observe:

i) The DMRG data presented in Fig. 1 and Fig. 5 (open circles) show a phase diagram divided in two regions well separated by a critical magnetic field, which depends on $J_{z}$ and which is around $h_{x} \simeq 2$ for $J_{z} \simeq 1$ in agreement with the isotropic case [20].

For $h_{x}$ below the critical magnetic field the system appears gapless. For $h_{x}$ above the critical magnetic field, a linear gap opens corresponding to the classical ferromagnetic phase, polarized along $x$-direction.

ii) As it appears in the Fig. 1 and Fig. 5 , where we plot the spin-wave theory gap (open diamonds), there is a substantial disagreement between our DMRG-simulation and SWT prediction [10].

iii) We find that the induced magnetization $M_{x}$ saturate for $h_{x}$ larger than the critical field, as illustrated in Fig. 6 for $J_{z}=0.25$ and $J_{z}=0.9706$ (and the staggered magnetization reduces to zero [21]). This explains the opening of a gap linear in $h_{x}$ in this phase.

Dmitriev et al have used scaling arguments 17] and found for the Hamiltonian (2) that a gap $\Delta \sim\left(h_{x}\right)^{\nu}$ 


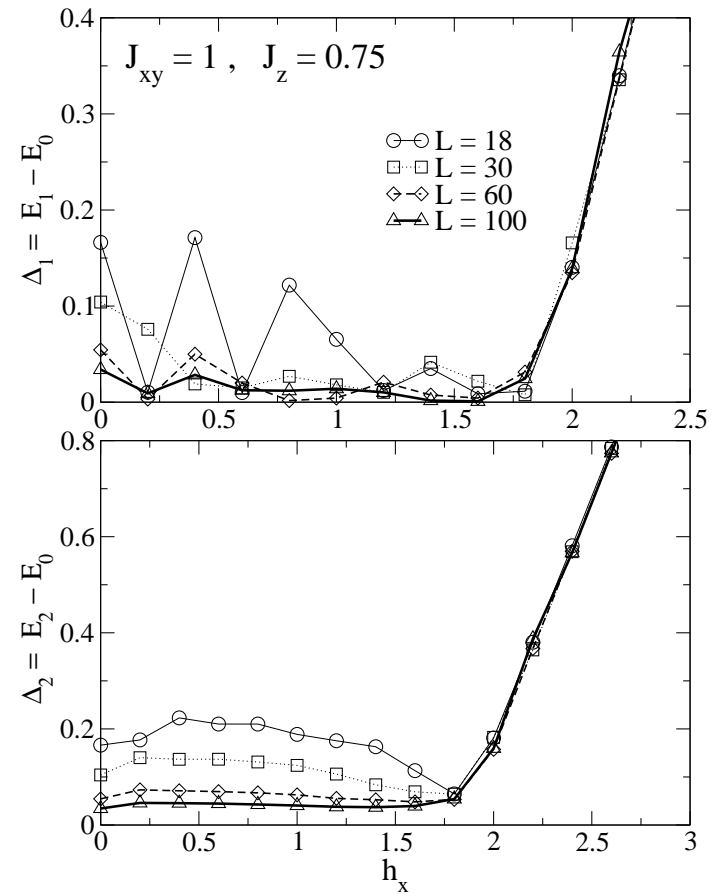

Fig. 2. Finite-size gaps observed for the Heisenberg chain of size $L$ in a transversal magnetic field $h_{x}$ with $J_{x y}=1, J_{z}=$ 0.75 .

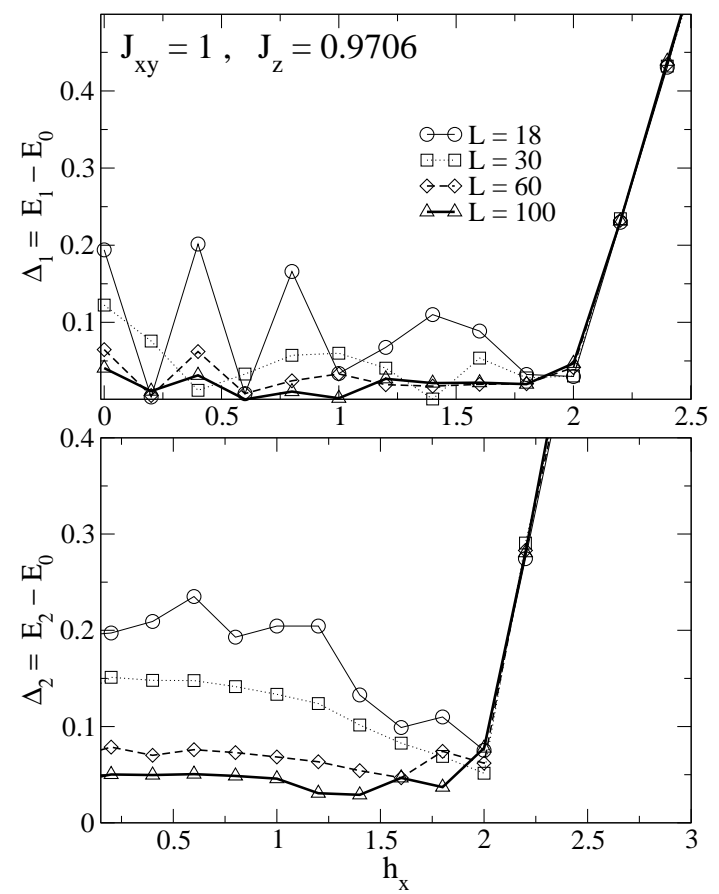

Fig. 3. Finite-size gaps observed for the Heisenberg chain of size $L$ in a transversal magnetic field $h_{x}$ with $J_{x y}=1, J_{z}=$ 0.9706 .

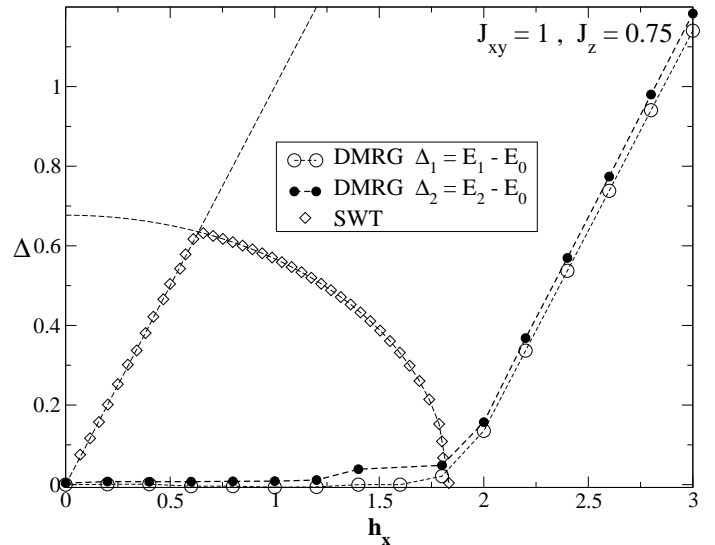

Fig. 4. The gap observed for the Heisenberg chain in a transversal magnetic field $h_{x}$ with $J_{x y}=1, J_{z}=0.75$. The circle symbols come from DMRG simulations $(m=50)$ extrapolated to the thermodynamic limit. The diamond symbols represent induced gap calculated from spin-wave theory.

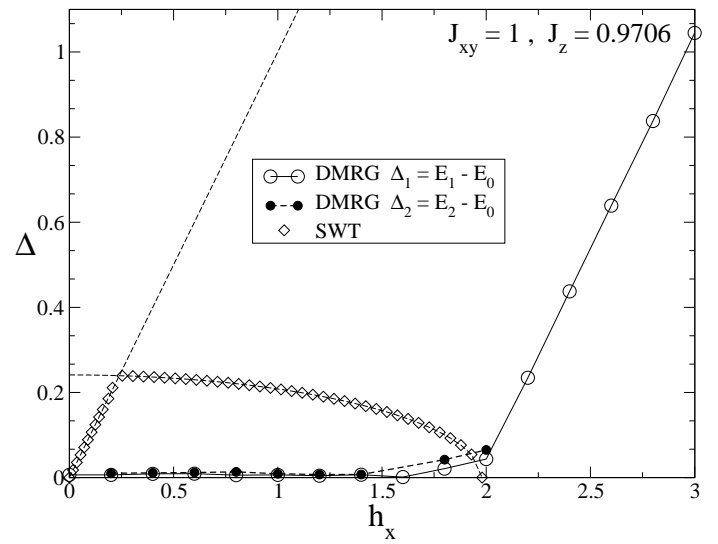

Fig. 5. The gap observed for the Heisenberg chain in a transversal magnetic field $h_{x}$ with $J_{x y}=1, J_{z}=0.9706$. The circle symbols come from DMRG simulations $(m=50)$ extrapolated to the thermodynamic limit. The diamonds represent the induced gap calculated from spin-wave theory.

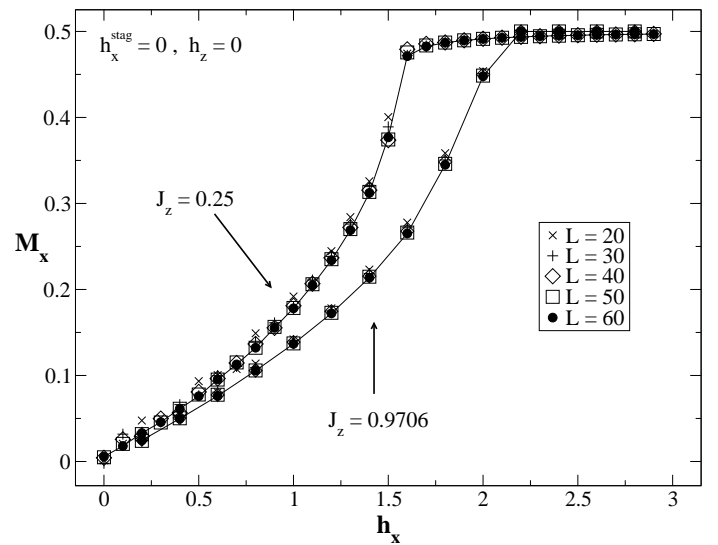

Fig. 6. The magnetization $M_{x}$ along $x$ for a anisotropy $J_{z}=$ $0.25,0.9706$. Note the saturation of $M_{x}$ above a certain critical field. 
opens for small external magnetic field, with an exponent $\nu$ depending on the anisotropy. Our simulations for small anisotropy are not supporting these predictions [17,22]. But due to the difficulty to resolve accurately $\Delta_{2}$ using DMRG, we cannot exclude completely an eventual small gap. Although till now we are not able to give a clear answer to the gap-opening question, but at least it seems that if there is a gap, the prefactor of the scaling law would need to be very small and in any case too small to explain experimental results on $\mathrm{Yb}_{4} \mathrm{As}_{3}$.

\section{Staggered magnetic field}

\subsection{Model}

The staggered Dzyaloshinsky-Moriya interaction, which is allowed [6, 7, 11] in the $4 f$-compound $\mathrm{Yb}_{4} \mathrm{As}_{3}$, leads in an external homogeneous magnetic field $h_{z}$ to an effective transversal staggered field $h_{x}^{\text {stag }}$. In this context the anisotropy is not relevant and we can consider the (isotropic) Heisenberg case $J_{x y}=J_{x} \equiv J$ :

$$
H=J \sum_{i=1}^{L-1} \mathbf{S}_{\mathbf{i}} \cdot \mathbf{S}_{\mathbf{i}+\mathbf{1}}+h_{x}^{\text {stag }} \sum_{i=1}^{L}(-1)^{i} S_{i}^{x}-h_{z} \sum_{i=1}^{L} S_{i}^{z} .
$$

The above Hamiltonian is not invariant under reflection with respect to the mid point of the chain when $L$ is even. However in the standard implementation of the DMRG algorithm, $L$ is even and the reflection symmetry is used. The Hamiltonian (4) can be easily made invariant under reflection by means of a local rotation, given by:

$$
\left(\begin{array}{c}
(-1)^{i} S_{i}^{x} \\
(-1)^{i} S_{i}^{y} \\
S_{i}^{z}
\end{array}\right) \rightarrow\left(\begin{array}{c}
S_{i}^{x} \\
S_{i}^{y} \\
S_{i}^{z}
\end{array}\right)
$$

The transformed Hamiltonian reads:

$$
\begin{gathered}
H=J \sum_{i=1}^{L-1}\left[S_{i}^{z} S_{i+1}^{z}-\left(S_{i}^{x} S_{i+1}^{x}+S_{i}^{y} S_{i+1}^{y}\right)\right] \\
+h_{x}^{\text {stag }} \sum_{i=1}^{L} S_{i}^{x}-h_{z} \sum_{i=1}^{L} S_{i}^{z}
\end{gathered}
$$

\subsection{Effect of the staggered transversal field}

We start by analyzing the finite-size dependence of the energy gap. The Fig. 7 shows the behavior of the finite size system gap versus the staggered magnetic field. One can clearly observe (i) that the gap vanishes in the thermodynamic limit only for zero $h_{x}^{\text {stag }}$ and (ii) that the magnetic correlation length is large but finite in the gapped case $h_{x}^{\text {stag }}>0$; the data for larger system size $L$ is essentially flat for $h_{x}^{\text {stag }}>0$.

In Fig. 8 we show the magnetization along the $z$-direction as a function of $h_{z}$, for various $h_{x}^{\text {stag }}$. Since $J_{x y}=$

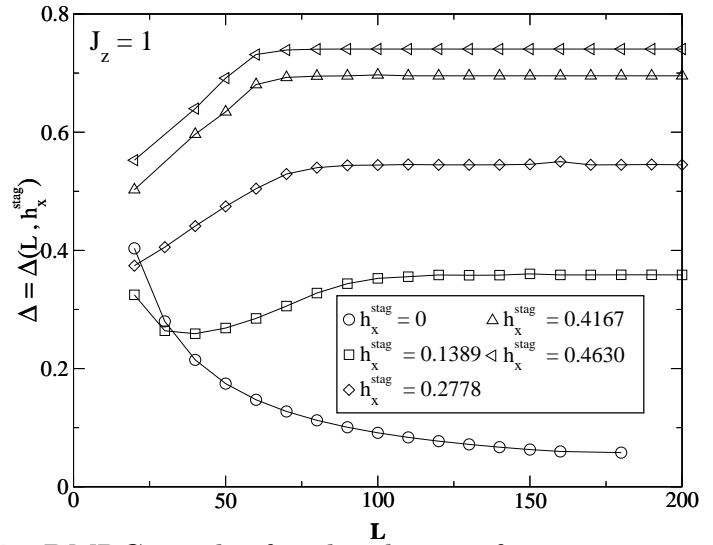

Fig. 7. DMRG results for the the gap for various staggered fields $h_{x}^{\text {stag }}$, as a function of chain length $L$, for $h_{z}=0$.

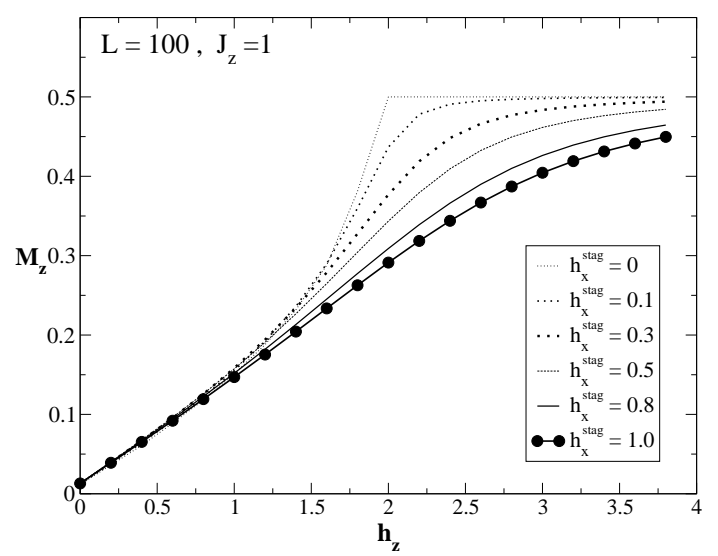

Fig. 8. Magnetization curves along the $z$-direction, compare 14 .

$J_{x} \equiv 1$ in (5), these results can be compared directly to those given in Fig. 6 for fields along $x$-direction. We note that the second-order phase transition to a completely magnetized state occurring at $h_{z}=2$ in the absence of a staggered field is progressively smeared out by $h_{x}^{\text {stag }}$; the transversal field induces quantum fluctuations into the magnetized state. These results confirm a similar study [14.

\subsection{Comparison with experiment}

Now using the experimental estimation for exchange coupling in the isotropic Heisenberg chain $J \simeq 26 \mathrm{~K}$, we fit the experimental data for the gap. The staggered magnetic field $h_{x}^{\text {stag }}$ is proportional to the experimental magnetic field $H^{\text {ext }}$ via $h_{x}^{\text {stag }}=c_{0} \sin (\theta) H^{\text {ext }}=c_{0} / g_{\perp} \sin (\theta) h_{z}$, where $g_{\perp}$ is g-factor for a magnetic-field perpendicular to the chain-direction. The g-factors for $\mathrm{Yb}_{4} \mathrm{As}_{3}$ are very anisotropic, $g_{\perp}$ has been estimated to be [14] $g_{\perp} \approx 1.3$.

Our fit yields $c_{0} / g_{\perp} \sin (\theta) \simeq 0.18$. The fitting to experimental data depends on $c_{0}$ which has not yet been determined precisely by experiment. While in the literature [11] $c_{0} \simeq 0.27$, we get a good agreement with our DMRG simulations and the experimental curve for $c_{0} \simeq 0.23$ (assuming $\left.g_{\perp} \simeq 1.3\right)($ Fig 9 ). 


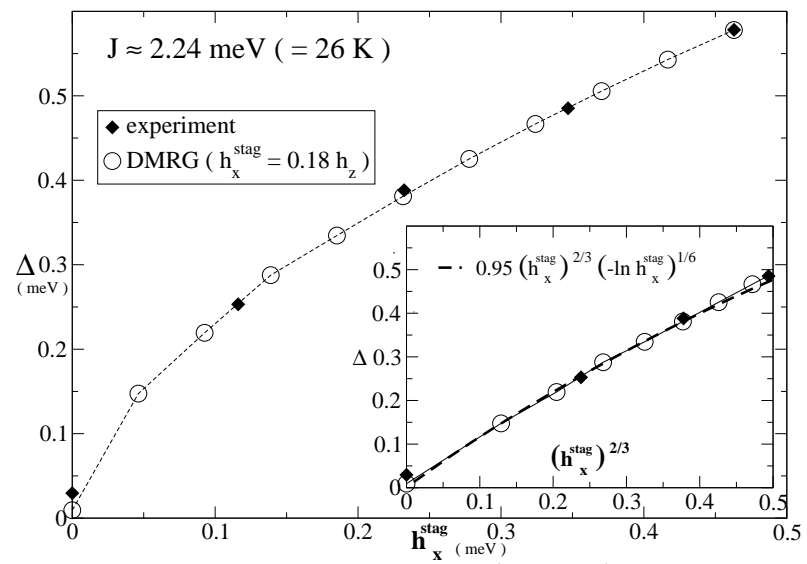

Fig. 9. Fit to experiment with data $(m=70)$ extrapolated to the thermodynamic limit.

\subsection{General case}

We consider now the case where the staggered and the uniform magnetic field are not proportional. We want to explore the excitation gap of

$H=\sum_{i=1}^{L-1}\left(\mathbf{S}_{\mathbf{i}} \cdot \mathbf{S}_{\mathbf{i}+\mathbf{1}}-\delta S_{i}^{z} S_{i+1}^{z}\right)+h_{x}^{\text {stag }} \sum_{i}^{L}(-1)^{i} S_{i}^{x}-h_{z} \sum_{i}^{L} S_{i}^{z}$.

In Fig. 10 we present for $h_{z}=0$ the gap $\Delta$ as a function of the anisotropy $\delta$ and the parameters $a_{0}$ and $a_{1}$ entering in the scaling-law

$$
\Delta=a_{0}\left(h_{x}^{\text {stag }}\right)^{a_{1}}
$$

for the gap. Bosonization predicts [12, 13] $a_{0} \approx 1.85$ and $a_{1}=2 / 3$ for the isotropic case $\left(\delta=0, h_{z}=0\right)$ for small staggered magnetic field $h_{x}^{\text {stag }}$. For the isotropic case $(\delta=$ 0 ) we present in Fig. 11 the same data as a function of $h_{z}$.

We also examined the DMRG-data using (7) including multiplicative logarithmic corrections [12, 13]: $\Delta=$ $a_{0}\left(h_{x}^{\text {stag }}\right)^{a_{1}}\left|\log h_{x}^{\text {stag }}\right|^{1 / 6}$ We found essentially the same values for the parameters $a_{0}$ and $a_{1}$ as presented in Fig. 10 and Fig. 11. We note, that (7) hold only in the asymptotic limit $h_{x}^{\text {stag }} \rightarrow 0$, which we do not examine in the present study. The results presented in Fig. 10 and Fig. 11 show $a_{0}$ and $a_{1}$ as obtained for overall fits to the gap, for $h_{x}^{\text {stag }} \leq 0.8$. We believe this parameter-region to be experimentally relevant.

\section{Conclusions}

We have studied the anisotropic Heisenberg-chain with staggered and uniform transversal and uniform longitudinal field by DMRG. We found no evidence for a substantial gap opening for a homogeneous transversal field, as predicted by a mean-field [10] and a scaling [17] analysis. We found, however, a gap opening for a staggered transversal field, in accordance with previous studies [12,13]. These results lead to the conclusion, that the gap-opening in

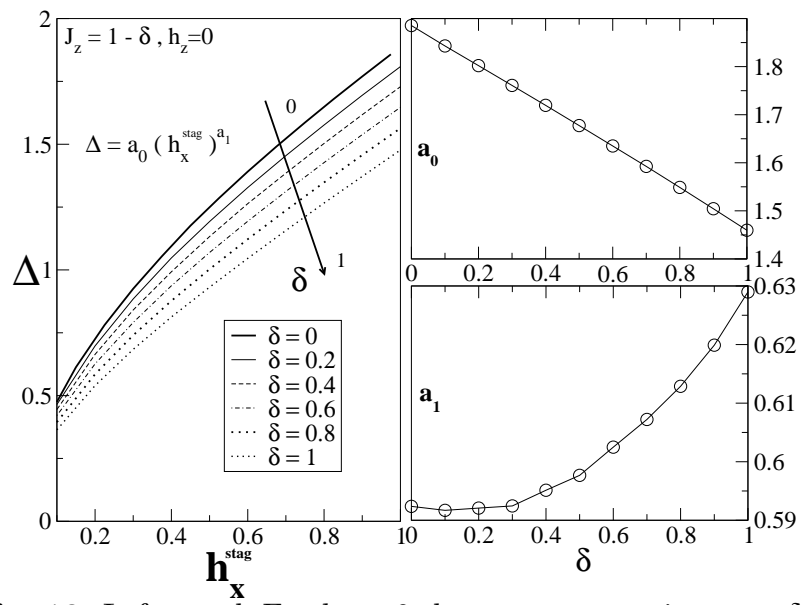

Fig. 10. Left panel: For $h_{z}=0$ the gap versus anisotropy $\delta$ in the thermodynamic limit $(m=40)$. Right panels: Dependence of the parameters $a_{0}$ and $a_{1}$ as a function of $\delta$.
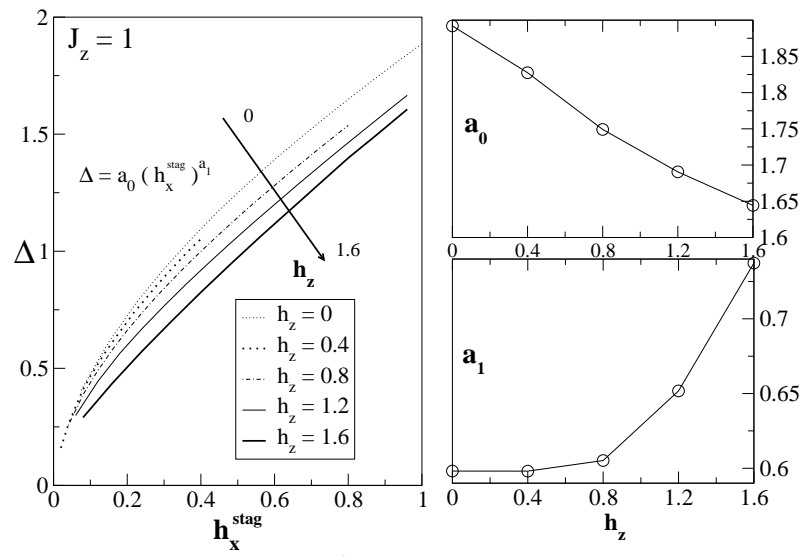

Fig. 11. Left panel: For $\delta=0$ the gap versus uniform longitudinal field $h_{z}$ in the thermodynamic limit $(m=40)$. Right panels: Dependence of the parameters $a_{0}$ and $a_{1}$ as a function of $\delta$.

the the rare-earth, $4 f$-compound $\mathrm{Yb}_{4} \mathrm{As}_{3}$ compound in an external magnetic field is attributable to a staggered Dzyaloshinsky-Moriya tensor [11, 6, [7].

\section{References}

1. B. S. Shastry and B. Sutherland, Phys. Rev. Lett. 47, 964 (1981).

2. H. Kageyama, K. Yoshimura, R. Stern, N. V. Mushnikov, K. Onizuka, M. Kato, K. Kosuge, C. P. Slichter, T. Goto and Y. Ueda, Phys. Rev. Lett. 82, 3168 (1999).

3. B. Schmidt, P. Thalmeier and P. Fulde, Euro. Phys. Lett. 35, 109 (1996).

4. A. Ochiai, T. Suzuki and T. Kasuya, J. Phys. Soc. Jpn. 59, 4129 (1990).

5. T. Kasuya, J. Phys. Soc. Jpn. 63, 2481 (1994).

6. H. Aoki, A. Ochiai, M. Oshikawa and K. Ueda, Physika B 281-282, 465 (2000).

7. H. Shiba, K. Ueda and O. Sakai, J. Phys. Soc. Jpn. 69, 1493 (2000). 
8. M. Kohgi, K. Iwasa, J.-M. Mignot, B. Fåk, P. Gegenwart, M. Lang A. Ochiai, H. Aoki and T. Suzuki, Phys. Rev. Lett. 86, 2439 (2001).

9. R. Helfrich, M. Kïoppen, M. Lang F. Steglich and A. Ochiai, J. Magn. Mat. 177-181, 309 (1998).

10. G. Uimin, Y. Kudasov, P. Fulde and A. Ovchinnikov, Eur. Phys. J. B 16, 241 (2000).

11. M. Oshikawa, K. Ueda, H. Aoki, A. Ochiai and M. Kohgi, J. Phys. Soc. Jpn. 68, 3181 (1999).

12. M. Oshikawa and I. Affleck, Phys. Rev. Lett. 79, 2883 (1997).

13. I. Affleck and M. Oshikawa, Phys. Rev. B 30, 1038 (1999).

14. H. Shibata and K. Ueda, J. Phys. Soc. Jpn 70, 3690 (2001).

15. S.R. White, Phys. Rev. Lett. 69, (1992) 2863 ; Phys. Rev. B 48, (1993) 10345.

16. S. Mori, J.-J. Kim and I. Harada, J. Phys. Soc. Jpn. 64, 3409 (1995).

17. D.V. Dmitriev, V.Ya. Krivnov, A.A. Ovchinnikov and A. Langari, cond-mat/0204590.

18. H. A. Bethe, Z. Phys. 71, 205 (1931).

19. F. Alcaraz, M. Barber, M. Batchelor, R. Baxter and G. Quispel, J. Phys. A: Math. Gen. 20, 6397 (1987).

20. R.B. Griffiths, Phys. Rev. 133, (1964) A768.

21. Y. Hieida, K. Okunishi and Y. Akutsu, cond-mat/0103371.

22. F. Essler, private communication. 